\title{
Buying Down the Cost of Direct Air Capture
}

\section{Supporting Information}

\author{
Klaus S Lacknera, Habib Azarabadia \\ a School of Sustainable Engineering \& the Built Environment, Arizona State University, Tempe, AZ 85287, \\ United States \\ *Corresponding Author, Email: klaus.lackner@asu.edu
}




\section{Section 1}

Approximating Sigma with Integral in Learning Curve Calculations

The accuracy of the approximation elaborated in Equation 7 is shown graphically in the figure below. As shown, for the range of $n$ (number of DAC farms) considered in this study, the integral's solution is sufficiently close to the sum result.

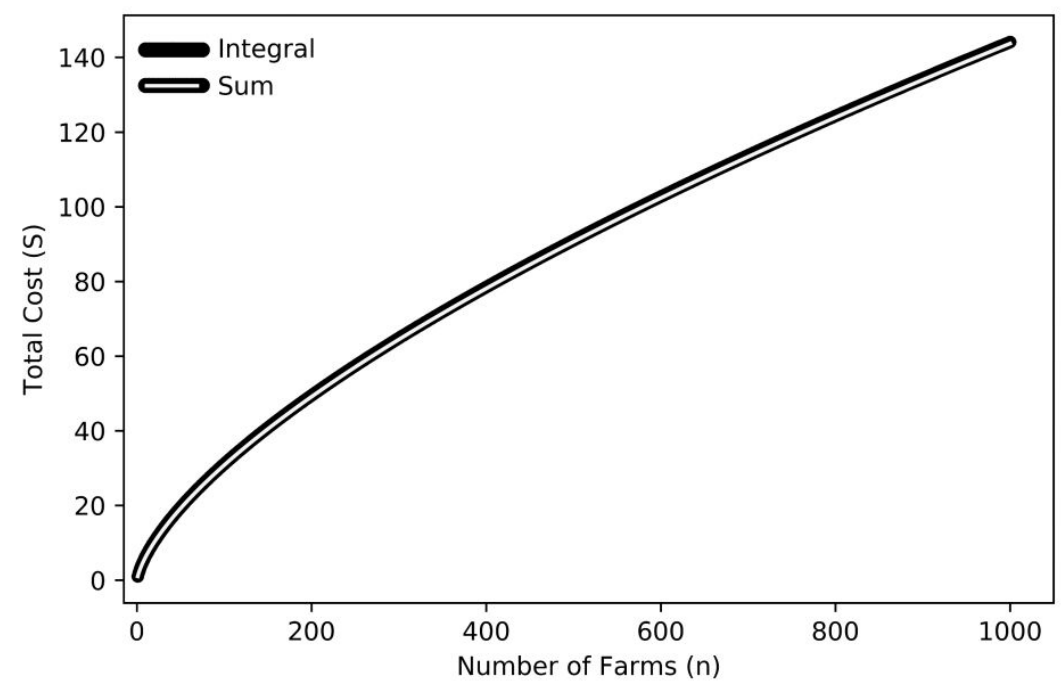

Figure S1 numerical difference between the sigma and integral in Equation 7 


\section{Section 2}

\section{Impact of Existing Units on Learning Curve Calculations}

The reducible cost component in Equation 1 is a function of implemented capacity $(n)$ based on the learning-by-doing empirical curve:

$$
c(n)=c_{1} n^{b} \quad \text { where } \quad \mathrm{b}=\log _{2} \varepsilon
$$

In our model, $c_{1}$ is the reducible cost right at the beginning of learning which is assumed $\$ 470$ per ton $\mathrm{CO}_{2}$. The current form of Equation $\mathrm{S} 1$ does not account for the existing DAC capacity which affects the number of doublings and the model results. To add this correction to the model, the relationship between $c_{1}$ and the initial DAC reducible cost before the implementation of the existing capacity $(E)$ can be shown as:

$$
c_{1}=c_{0} E^{b}
$$

Parameter $c_{0}$ is the initial reducible cost before the existing capacity. Starting from the very beginning of DAC implementation (before the existing capacity), Equation S1 can be rewritten:

$$
c(n)=c_{0}(E+n)^{b}
$$

And by substituting from Equation S2 we will have:

$$
c(n)=\frac{c_{1}}{E^{b}}(E+n)^{b}
$$

Equation S4 is the corrected form of the learning curve equation by accounting for the existing capacity. As a result, the number of farms to reach the target price (Equation 10) can be driven based on Equation S4:1

$$
n_{t}=E\left[\left(\frac{t-r}{c_{1}}\right)^{\frac{1}{b}}-1\right]
$$

The only problem with this equation is that it does not allow the simple analytical development of the rest of the model i.e., Equations 12,13 , and 15 . To solve this issue, we can keep the general form of the reducible cost equation while replacing $c_{1}$ with a corrected value to account for the existing capacity $(E)$. By defining the corrected value $\left(c_{1}^{\prime}\right)$, we can use the short

\footnotetext{
${ }^{1}$ Instead of $\alpha$ in Equation 10, we have used parameter $b$ in Equation S5. $\alpha=1+b$
} 
and simple analytical model developed in the paper while accounting for the existing capacity. The corrected value must satisfy the following equation:

$$
c(n)=c_{1}^{\prime} n^{b}=\frac{c_{1}}{E^{b}}(E+n)^{b}
$$

We can rewrite the previous equation:

$$
c_{1}^{\prime}=\frac{c_{1}}{E^{b}}\left(\frac{E+n}{n}\right)^{b}
$$

In a cost projection where the accumulating capacity is significantly larger than the existing capacity $(n \gg E)$, the second ratio on the right side of Equation S7 will be very close to 1 . Therefore:

$$
c_{1}^{\prime} \approx \frac{c_{1}}{E^{b}}
$$

As a result, by replacing the $c_{1}$ value with $\frac{c_{1}}{E^{b}}$ in all equations, the current model can be corrected to account for the existing capacity before the beginning of learning calculations. Table S1 demonstrates the model sensitivity to the $E$ value and challenges the accuracy of our approximation. Assuming 4 different values for the existing capacity, we compare the accurate method (Equation S5) with our approximation (correcting the $c_{1}$ value with Equation S8 and using Equation 10). As shown in the table, in small existing capacities the accuracy of our approximation is satisfying.

The results in the paper are calculated based on $E=1$.

Table S1 The accuracy of the approximation used in including the existing capacity (E) in the learning curve calculations.

\begin{tabular}{|c|c|c|c|c|c|}
\hline \multirow{2}{*}{ Threshold Value } & \multirow{2}{*}{ Calculation } & \multicolumn{4}{|c|}{$c_{1}=\$ 470 /$ ton } \\
\cline { 3 - 6 } & Method & $E=1$ & $E=2$ & $E=10$ & $E=100$ \\
& & $c_{1}^{\prime}=\$ 470 /$ ton & $c_{1}^{\prime}=\$ 600 /$ ton & $c_{1}^{\prime}=\$ 1000 /$ ton & $c_{1}^{\prime}=\$ 2250 /$ ton \\
\hline \multirow{2}{*}{ No. of Farms $\left(n_{t}\right)$} & Eq. S5 & 302 & 603 & 3,016 & 30,169 \\
\cline { 2 - 6 } & Eq. S8 \& 10 & 303 & 614 & 3,171 & 3,3224 \\
\hline & & $0.3 \%$ & $1.7 \%$ & $5.1 \%$ & $10.1 \%$ \\
\hline
\end{tabular}




\section{Section 3}

Uncertainty Analysis Results for Number of Farms and Total Money Spent

Monte Carlo simulation results for the number of farms $\left(n_{t}\right)$ and total investment $\left(K_{\text {threshold }}\right)$. The vertical axis shows the cumulative probability curves (success probability) of reaching the target DAC cost based on the number of farms $\left(n_{t}\right)$ built and the total investment value ( $\left.K_{\text {threshold }}\right)$.

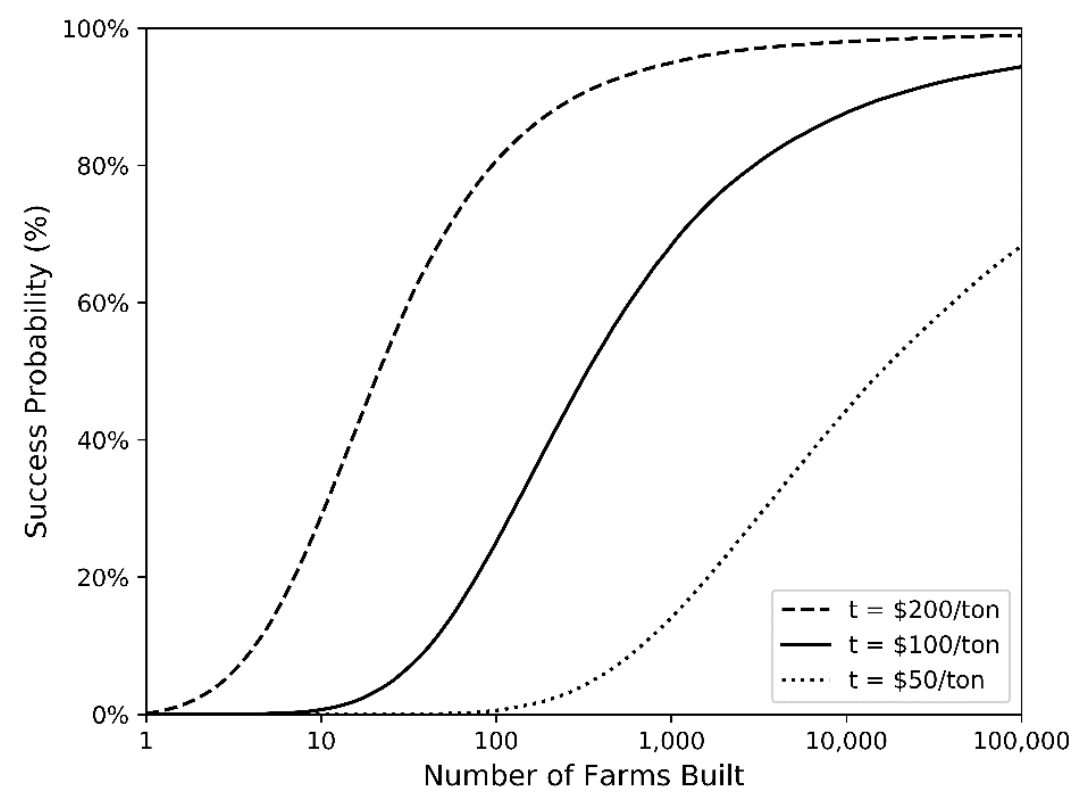

Figure S2 Cumulative probability function for successful DAC commercialization based on the number of farms built for three different target prices $\left(\$ 50, \$ 100\right.$, and $\$ 200$ per ton of $\left.\mathrm{CO}_{2}\right)$ 


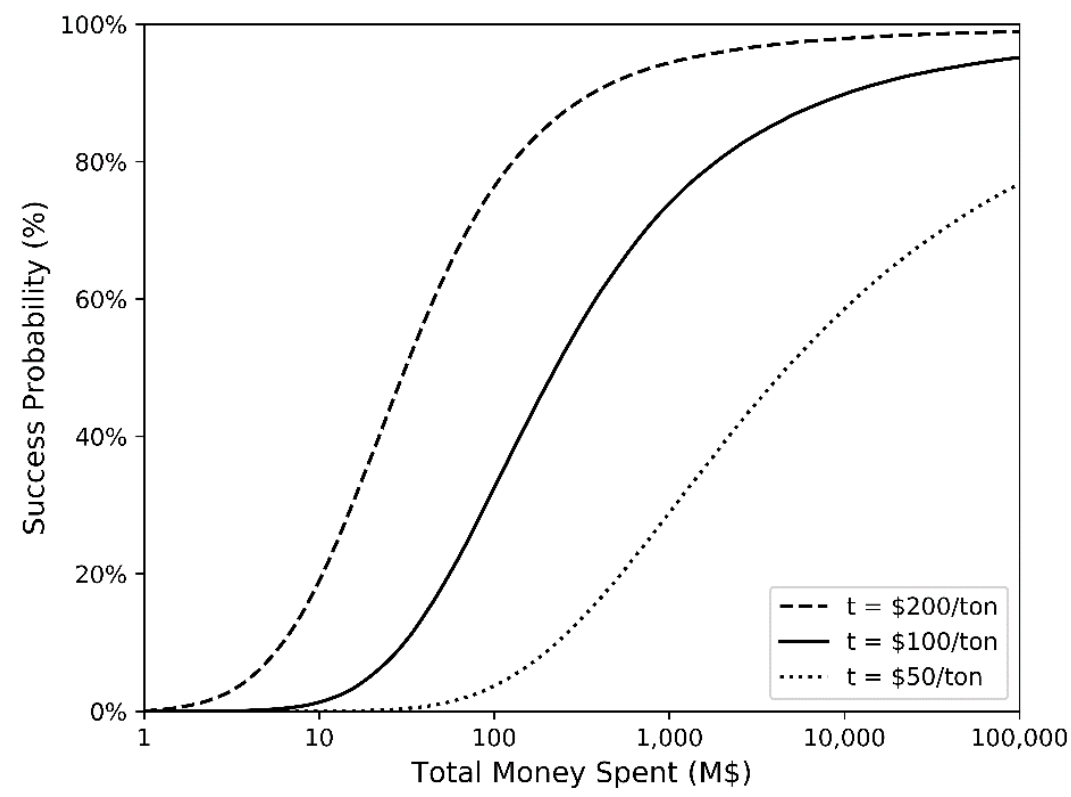

Figure S3 Cumulative probability function for successful DAC commercialization based on the total amount of investment for three different target prices $\left(\$ 50, \$ 100\right.$, and $\$ 200$ per ton of $\left.\mathrm{CO}_{2}\right)$ 